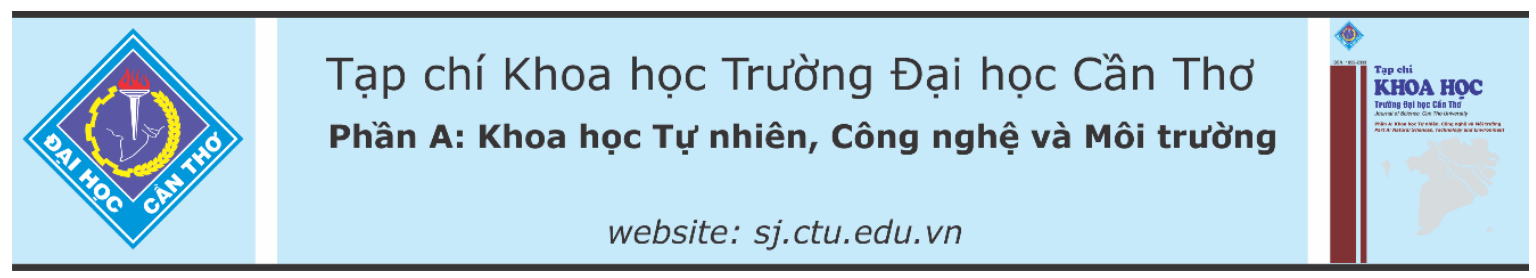

DOI:10.22144/ctu.jvn.2021.002

\title{
XÁC ĐỊNH NHÂN TỐ ẢNH HƯởNG ĐẾN GIÁ ĐẤT TRÊN ĐỊA BÀN THÀNH PHỐ CẦN THƠ
}

Phạm Thanh Thảo ${ }^{1 *}$ và Phan Trung Hiền ${ }^{2}$

${ }^{1} U B N D$ phuoòng An Bình, quận Ninh Kiều, thành phố Cần Tho

${ }^{2}$ Khoa Luật, Truoòng Đại học Cần Tho'

*Nguoòi chịu trách nhiệm về bài viết: Phạm Thanh Thảo (email: phamthanhthao8889@ gmail.com)

\section{Thông tin chung:}

Ngày nhận bài: 19/11/2020

Ngày nhận bài sưa: 07/01/2021

Ngày duyệt đăng: 27/03/2021

\section{Title:}

Determination of factors affecting land price in Can Tho city

\section{Tù khóa:}

Giá đất, định giá đất, xác định yếu tố, yếu tố ảnh hưởng

\section{Keywords:}

Determination of factors, factors affecting land prices, land value

\begin{abstract}
The study was conducted to determine the factors that affect land price in Can Tho city based on the application of exploratory factor analysis model (EFA) and multivariate linear regression model. The survey was conducted with 328 land users in Can Tho city during the period from June to December 2019. The results of multivariate linear regression analysis show that $61.0 \%$ of the variation in land price is affected by groups of factors included in the research model, while the rest $(39,0 \%)$ was explained by other factors outside the model. The obtained results showed that there were six groups of factors affecting the land prices in Can Tho city, including: natural factor group , other factor group, economic factor group, group of factors of infrastructure, group of legal factors, State policy, group of social factors. The group of natural factors had the highest effect on land price with 12,86\% contribution and social factors had the least effect on land price with contribution $6,45 \%$.
\end{abstract}

\section{TÓM TẮT}

Nghiên cứu được thực hiện với muc đích xác định các nhân tố ảnh hưởng đến giá đất trên địa bàn thành phố Cần Thơ trên cơ sở ưng dụng mô hình phân tích nhân tố khám phá (Exploratory Factor Analysis - EFA) và mô hình hồi quy tuyến tính đa biến. Điều tra được thực hiện với 328 người sủ dụng đất trên địa bàn thành phố Cần Tho trong thời gian tù̀ tháng 6 12/2019. Kết quả phân tích hồi quy tuyến tính đa biến cho thấy $61,0 \%$ sư biến động của giá đất bị ảnh huơong bởi các nhóm yếu tố đưa vào mô hình nghiên cứu, còn lai 39,0\% sư biến động của giá đất là do các yếu tố khác. Kết quả nghiên cứu có 6 nhóm yếu tố có ảnh hưởng tích cưc đến giá đất tại thành phố Cần Tho gồm: nhóm nhân tố tư nhiên, nhóm nhân tố khác, nhóm nhân tố kinh tế, nhóm nhân tố ha tầng, nhóm nhân tố pháp lý, chính sách Nhà nuớc, nhóm nhân tố xã hội. Trong đó nhóm nhân tố tự nhiên được xác định là có ảnh huơong lớn nhất đển giá đất với sụ đóng góp 12,86\%, nhóm nhân tố xã hội được xác định là it ảnh hương đến giá đất nhất với sự đóng góp $6,45 \%$. 


\section{1. ĐẶT VẤN ĐỀ}

Đô thị hóa ở Việt Nam đang trong thời kì phát triển mạnh mẽ, đặc biệt là ở các thành phố lớn như: Hà Nội, thành phố Hồ Chí Minh, Đà Nẵng và Cần Thơ. Đô thị hóa làm cho nhu cầu đất đai để xây dựng các công trình phục vụ xã hội, công trình nhà ở, trung tâm thương mại ngày càng tăng lên nhưng quỹ đất thì có hạn, điều này vô hình chung đẩy giá đất ngày càng tăng cao. Bên cạnh đó là tình trạng đầu cơ đất đai, môi giới vì lợi ích cá nhân đã đưa các thông tin ảo để đẩy giá đất ngày càng lên cao. Vì thế, những người có thu nhập thấp rất khó tiếp cận nguồn tài nguyên khan hiếm này. Theo $\mathrm{Vu}$ and Kawashima (2018), giá đất đai tại các thành phố lớn Việt nam đều ngang bằng với giá đất tại khu dân cư ở một số địa điểm ở trung tâm của Tokyo, mặc dù thủ đô Nhật Bản và Việt Nam có khoảng cách lớn. Vấn đề đặt ra là làm sao để có thể xác định được chính xác giá đất theo đúng với giá trị của nó. Thật sự điều này khó có thể thực hiện do đất đai là một loại hàng hóa đặc biệt, không giống các hàng hóa khác ở chỗ đất đai là có hạn, giá trị của đất đai không giống nhau do giá trị đất đai bị ảnh hưởng bởi nhiều yếu tố khác nhau. Việc nghiên cứu xác định các yếu tố ảnh hưởng đến giá đất đã được nhiều nhà nghiên cứu thực hiện từ rất lâu. Đa số các kết quả nghiên cứu cho kết quả yếu tố vị trí và diện tích là hai yếu tố có ảnh hưởng mạnh mẽ nhất đển giá đất (Northcraft and Small, 1979, Reynolds et al.,1978). Theo Phan Thị Thanh Huyền và ctv. (2017) chỉ ra có 4 nhóm yếu tố có ảnh hưởng đến giá đất ở đô thị trên địa bàn thành phố Điện Biên Phủ, tỉnh Điện Biên là nhóm yếu tố xã hội, nhóm yếu tố kinh tế, nhóm yếu tố khu vực và nhóm các yếu tố riêng biệt.

Thành phố Cần Thơ được xem là trung tâm của Đồng bằng sông Cửu Long, là trung tâm kinh tế, là đầu mối quan trọng về giao thông vận tải nội vùng và liên vận quốc tế; đóng vai trò động lực thúc đẩy mạnh mẽ sự phát triển của toàn vùng Đồng bằng sông Cửu Long. Với vị trí quan trọng này, trong những năm qua thành phố Cần Thơ đã không ngừng phấn đất để đạt được các mục tiêu đề ra. Bên cạnh đó, giá đất trên địa bàn cũng tăng tỷ lệ thuận với sự phát triển của thành phố và có sự chênh lệch lớn giữa giá đất do Nhà nước quy định và giá đất trên thị trường, có khu vực khoảng cách này chênh lệch đến $7-8$ lần. Mục đích của nghiên cứu này là xác định các yếu tố ảnh hưởng đến giá đất tại thành phố Cần Thơ trên cơ sở ứng dụng mô hình phân tích nhân tố khám phá (Exploratory Factor Analysis - EFA) và áp dụng mô hình hồi quy tuyến tính đa biến.

\section{PHƯƠNG PHÁP NGHIÊN CÚU}

\subsection{Giả thuyết}

Dựa trên kết quả các nghiên cứu của Hồ Thị Lam Trà, 2005, Phan Thị Thanh Huyền và ctv., (2017), Phạm Phương Nam và ctv. (2019) và đặc điểm của địa điểm nghiên cứu, mô hình nghiên cứu đã được xây dựng (Hình 1). Tất cả các nhóm nhân tố tự nhiên, nhóm nhân tố khác, nhóm nhân tố kinh tế, nhóm nhân tố hạ tầng, nhóm nhân tố pháp lý, chính sách Nhà nước, nhóm nhân tố xã hội được giả định có ảnh hưởng tích cực đến giá đất trên địa bàn thành phố Cần Thơ.

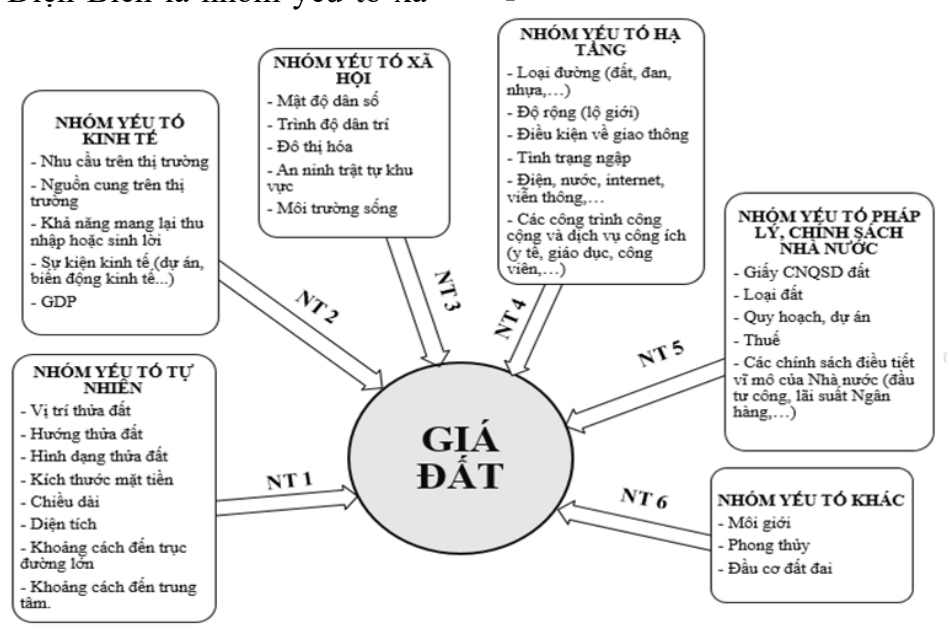

Hình 1. Mô hình nghiên cứu

\subsection{Phương pháp}

\subsubsection{Thu thập dĩ liệu}

Số liệu thứ cấp về bản đồ, hiện trạng sử dụng đất, tài liệu thống kê, báo cáo, và giá đất của thành phố Cần Thơ được thu thập từ Sở Tài nguyên và Môi trường và Ủy ban nhân dân thành phố Cần Thơ. 
Số liệu sơ cấp được thu thập dưới dạng phản hồi từ những người được hỏi. Cỡ mẫu được xác định dựa trên các yêu cầu của phân tích nhân tố khám phá và phân tích Hồi quy (Hoàng Trọng và Chu Nguyễn Mộng Ngọc, 2005). Số lượng mẫu tối thiểu của nghiên cứu là 150 người tham gia. Tuy nhiên, dựa trên số lượng chuyển nhượng quyền sử dụng đất trên địa bàn thành phố Cần Thơ, nghiên cứu đã tiến hành điều tra 328 người tham gia ( 90 cán bộ, công chức, viên chức làm công tác quản lý đất đai, 81 nhân viên ngân hàng, công chứng viên, 23 công nhân, nông dân, 11 cán bộ hưu trí, 01 học sinh, sinh viên và 122 khác (môi giới, nhân viên văn phòng)) được thực hiện từ tháng $6 / 2019$ đến tháng 12/2019 để thu thập các số liệu về thực trạng giá đất giao dịch trên thị trường để phục vụ nghiên cứu của đề tài. Thang đo Likert 5 cấp từ 1 đến 5 được sử dụng để thiết kế bảng câu hỏi về đo lường ảnh hưởng của các yếu tố ảnh hưởng giá đất, trong đó $5=$ Rất đồng ý, $4=$ Đồng ý, $3=$ Trung hòa (Phân vân), $2=$ Không đồng ý và $5=$ Rất không đồng ý) (Likert, 1932). Người trả lời được yêu cầu chỉ chọn một phương án cho mọi câu hỏi. Hair et al. (2006) cho rằng để sử dụng phân tích nhân tố khám phá EFA, kích thước mẫu tối thiểu phải là 50 , tốt hơn là 100 và tỷ lệ quan sát/ biến đo lường là $5: 1$. Tương tự cỡ mâ̂u được xác định dựa trên các yêu cầu của phân tích nhân tố khám phá và phân tích hồi quy (Hoàng Trọng và Chu Nguyễn Mộng Ngọc, 2005), tức là số quan sát (cỡ mẫu) ít nhất phải bằng 4 hay 5 lần số biến quan sát trong phân tích nhân tố thì số liệu mới có ý nghĩa. Như vậy số lượng mẫu tối thiểu của nghiên cứu là 150 . Tuy nhiên, dựa trên số lượng chuyển nhượng quyền sử dụng đất tại 2 quận Ninh Kiều, Cái Răng và huyện Cờ Đỏ thành phố Cần Thơ, nghiên cứu đã điều tra 328 quan sát (138 quan sát tại quận Ninh Kiều, 139 quan sát tại quận Cái Răng và 51 quan sát tại huyện Cờ Đỏ).

\subsubsection{Phân tích dũ liệu}

Phân tích yếu tố khám phá (EFA) được sử dụng để xác định mối quan hệ cơ bản giữa các biến đo lường (Norris et al., 2010), kiểm định giá trị thang đo, thực hiện sau khi đánh giá độ tin cậy của thang đo bằng hệ số Cronbach's Alpha và loại đi các biến không đảm bảo độ tin cậy. Các thông số được áp dụng là:

- Các biến chỉ được chấp nhận khi KMO nằm trong khoảng [0,5-1]. Trị số KMO lớn có ý nghĩa phân tích yếu tố là thích hợp.
- Kiểm định Barlett's với mức ý nghĩa (Sig. < 0,05 ) để đảm bảo các yếu tố có mối tương quan với nhau trong tổng thể.

- Hệ số Eigenvalue có giá trị $>1$ để đảm bảo các nhóm yếu tố có sự khác biệt.

- Tổng phương sai giải thích: thang đo chỉ được chấp nhận khi tổng phương sai giải thích (Total variance explained) $>50 \%$.

- Factor loading $>0,3$ được xem là đạt mức tối thiểu; Factor loading $>0,4$ được xem là quan trọng; Factor loading $>0,5$ được xem là có ý nghĩa thực tiễn.

Để tiến hành phân tích nhân tố khám phá, trước hết là kiểm định độ tin cậy của thang đo thông qua kiểm định Cronbach's Alpha (Cronbach, 1951). Giá trị alpha tối đa 0,90 đã được đề xuất (Streiner, 2003). Dữ liệu sẽ được chấp nhận khi Tổng tương quan > 0,3 (Nunnally and Bernstein1994; Hair et al., 1998).

\section{KẾT QUẢ VÀ THẢO LUẬN}

\subsection{Xây dựng nhóm yếu tố ảnh hưởng đến giá đất tại thành phố Cần Thơ}

Có nhiều yếu tố ảnh hưởng đến giá đất: nhóm yếu tố khu vực (vị trí, hạ tầng, chất lượng môi trường, quy hoạch), yếu tố xã hội (an ninh và tỷ lệ tội phạm, tâm lý, tinh thần, thị hiếu, đô thị hóa, mật độ dân số, đầu cơ), các yếu tố kinh tế (tốc độ phát triển kinh tế, thu nhập và chi tiêu, biến động giá cả, lãi suất ngân hàng, dự trữ, đầu tư), nhóm yếu tố riêng lẻ (diện tích, chiều rộng mặt tiền, chiều sâu thửa đất, hình dạng đất, độ dốc, giới hạn quy hoạch đô thị), yếu tố pháp lý (giấy chứng nhận quyền sử dụng đất, quyết định giao đất ), các yếu tố quốc tế (kinh tế thế giới, chính trị thế giới), các yếu tố liên quan đến thị trường (cung cầu, quan hệ cung cầu), các yếu tố nhà nước và luật pháp (chính sách tài chính, tín dụng, chính sách thuế, chính sách đầu tư, chính sách đất đai) (Hồ Thị Lam Trà, 2005, Phan Thị Thanh Huyền và ctv. (2017)). Trên cơ sở đó nghiên cứu đã kế thừa đồng thời có bổ sung và điều chỉnh một số nhóm biến như nhóm yếu tố khác bao gồm (yếu tố phong thủy, yếu tố môi giới, yếu tố đầu cơ đất đai) được các chuyên gia cho rằng phù hợp với đặc trưng của thành phố Cần Thơ. Sáu nhân tố độc lập với 32 biến quan sát được chọn lựa để nghiên cứu. Đây là những yếu tố ảnh hưởng đáng kể đến giá đất ở Cần Thơ hiện nay (Bảng 1). Các yếu tố này có mối quan hệ chặt chẽ với sự biến động của thị trường bất động sản nói chung và thị trường đất đai nói riêng. 
Bảng 1. Nhân tố ảnh hưởng đến giá đất ở thành phố Cần Thơ

\begin{tabular}{|c|c|c|}
\hline Nhóm yếu tố & Ký hiệu & Diễn giải các yếu tố thuộc tính \\
\hline \multirow{8}{*}{ Tự nhiên } & TN1 & Kích thước mặt tiền \\
\hline & TN2 & Hình dạng thửa đất \\
\hline & TN3 & Khoảng cách đến trục đường lớn \\
\hline & TN4 & Chiều dài \\
\hline & TN5 & Vị trí thửa đất \\
\hline & TN6 & Diện tích thửa đất \\
\hline & TN7 & $\begin{array}{l}\text { Khoảng cách đến trung tâm (TTTM, TTHC, nơi làm việc, bệnh viện, trường } \\
\text { học...) }\end{array}$ \\
\hline & TN8 & Hướng thửa đất \\
\hline \multirow{5}{*}{ Kinh tế } & KT1 & Khả năng mang lại thu nhập hoặc sinh lời của thửa đất \\
\hline & KT2 & Sự kiện kinh tế (có dự án đầu tư, biến động kinh tế trong nước và quốc tế,...) \\
\hline & KT3 & Nhu cầu đất đai trên thị trường \\
\hline & KT4 & Nguồn cung đất đai trên thị trường \\
\hline & KT5 & Mức thu nhập bình quân của người dân \\
\hline \multirow{5}{*}{ Xã hội } & XH1 & Mật độ dân số khu vực quanh thửa đất \\
\hline & $\mathrm{XH} 2$ & Trình độ dân trí khu vực quanh thửa đất \\
\hline & XH3 & Đô thị hóa \\
\hline & XH4 & An ninh trật tự khu vực quanh thửa đất \\
\hline & XH5 & Chất lượng cảnh quan, môi trường khu vực quanh thửa đất \\
\hline \multirow{6}{*}{ Hạ tầng } & HT1 & Loại đường (đất, đan, nhựa,...) \\
\hline & HT2 & Tình trạng ngập \\
\hline & HT3 & Điều kiện về giao thông \\
\hline & HT4 & Các công trình công cộng và dịch vụ công ích (y tế, giáo dục, công viên,...) \\
\hline & HT5 & Độ rộng của đường (lộ giới) gắn với thửa đất \\
\hline & HT6 & Tình trạng điện, nước, internet, viễn thông... \\
\hline \multirow{5}{*}{$\begin{array}{l}\text { Pháp lý, chính } \\
\text { sách Nhà nước }\end{array}$} & PLCS1 & Thuế suất của thửa đât \\
\hline & PLCS2 & Quy hoạch của Nhà nước \\
\hline & PLCS3 & Loại đất \\
\hline & PLCS4 & Giấy tờ pháp lý của thửa đất \\
\hline & PLCS5 & $\begin{array}{l}\text { Các chính sách điều tiết vĩ mô của Nhà nước (đầu tư công, thu hút FDI, dự } \\
\text { án, lãi suất Ngân hàng,...) }\end{array}$ \\
\hline \multirow{3}{*}{$\begin{array}{l}\text { Nhóm yếu tố } \\
\text { khác }\end{array}$} & K1 & Môi giới \\
\hline & $\mathrm{K} 2$ & Đầu cơ đất đai \\
\hline & $\mathrm{K} 3$ & Phong thủy \\
\hline
\end{tabular}

\subsection{Kiểm tra độ tin cậy Cronbach's alpha và phân tích nhân tố khám phá EFA}

\subsubsection{Kiểm tra độ tin cậy Cronbach's alpha}

Kết quả phân tích Cronbach's Alpha có 6 nhóm yếu tố và 28 biến quan sát thỏa điều kiện, phù hợp với hệ số Cronbach's Alpha nằm trong khoảng $[0,6-$ $0,95]$ và có hệ số tương quan biến tồng (Corrected Item-Rest Correlation) lớn hơn 0,3 (Nunnally and Bernstein., 1994; Hair et al., 1998).

Bảng 2 cho thấy có 6 nhóm yếu tố và chỉ còn 28 biến quan sát thỏa điều kiện vì hệ số Cronbach's Alpha nằm trong khoảng $[0,6-0,95]$ và có hệ số tương quan biến tổng (Corrected Item-Rest Correlation) lớn hơn 0,3 (Nunnally and Bernstein, 1994; Hair et al., 1998). Điều này chứng tỏ tất cả các dữ liệu đều đảm bảo độ tin cậy.

Trong quá trình kiểm tra, có 4 yếu tố không phù hợp đó là Khoảng cách đến trung tâm (trung tâm thương mại, trung tâm hành chính, nơi làm việc, bệnh viện, trường học), Thu nhập bình quân của người dân, Loại đường (đất, đan, nhựa) và Tình trạng ngập do có hệ số tương quan tổng lần lượt là $0,119,0,287,0,257,0,184<0,3$. Điều này ngụ ý rằng 4 biến này không đủ điều kiện để phân tích thêm. 
Bảng 2. Kết quả kiểm định độ tin cậy thang đo biến độc lập

\begin{tabular}{|c|c|c|c|}
\hline Ký hiệu & Các tiêu chí đánh giá & $\begin{array}{r}\text { Hệ số tương quan } \\
\text { biến - tổng }\end{array}$ & $\begin{array}{l}\text { Cronbach's Alpha } \\
\text { nếu loại mọ̣t biến }\end{array}$ \\
\hline & Nhóm biến yếu tố tự nhiên (TN) & & $\mathbf{0 , 8 6 3}$ \\
\hline TN1 & Kích thước mặt tiền & 0,636 & 0,843 \\
\hline $\mathrm{TN} 2$ & Hình dạng thửa đất & 0,640 & 0,844 \\
\hline TN3 & Khoảng cách đến trục đường lớn & 0,579 & 0,851 \\
\hline TN4 & Chiều dài & 0,623 & 0,846 \\
\hline TN5 & Vị trí thửa đất & 0,731 & 0,829 \\
\hline TN6 & Diện tích thửa đất & 0,653 & 0,841 \\
\hline \multirow[t]{2}{*}{ TN7 } & $\begin{array}{l}\text { Khoảng cách đến trung tâm (TTTM, TTHC, nơi làm việc, } \\
\text { bệnh viện, trường học) }\end{array}$ & 0,605 & 0,849 \\
\hline & Nhóm biến yếu tố kinh tế (KT) & & 0,856 \\
\hline KT1 & Khả năng mang lại thu nhập hoặc sinh lời của thửa đất & 0,646 & 0,838 \\
\hline KT2 & $\begin{array}{l}\text { Sự kiện kinh tế (có dự án đầu tư, biến động kinh tế trong nước } \\
\text { và quốc tê) }\end{array}$ & 0,759 & 0,793 \\
\hline KT3 & Nhu cầu đất đai trên thị trường & 0,759 & 0,791 \\
\hline \multirow[t]{2}{*}{ KT4 } & Nguồn cung đất đai trên thị trường & 0,643 & 0,843 \\
\hline & Nhóm biến yếu tố xã hội (XH) & & 0,838 \\
\hline $\mathrm{XH} 1$ & Mật độ dân số quanh thửa đất & 0,569 & 0,824 \\
\hline $\mathrm{XH} 2$ & Trình độ dân trí quanh thửa đất & 0,572 & 0,826 \\
\hline XH3 & Đô thị hóa & 0,679 & 0,796 \\
\hline XH4 & An ninh trật tự khu vực & 0,683 & 0,794 \\
\hline \multirow[t]{2}{*}{ XH5 } & Chất lượng cảnh quan, môi trường khu vực quanh thửa đất & 0,711 & 0,786 \\
\hline & Nhóm biến yếu tố hạ tầng $(\mathrm{HT})$ & & 0,746 \\
\hline HT3 & Điều kiện về giao thông & 0,524 & 0,697 \\
\hline HT4 & $\begin{array}{l}\text { Các công trình công cộng và dịch vụ công ích (y tế, giáo dục, } \\
\text { công viên) }\end{array}$ & 0,555 & 0,679 \\
\hline HT5 & Độ rộng của đường (lộ giới) gắn với thửa đất & 0,561 & 0,676 \\
\hline \multirow[t]{2}{*}{ HT6 } & Tình trạng điện, nước, internet, viễn thông... & 0,520 & 0,699 \\
\hline & Nhóm yếu tố pháp lý, chính sách Nhà nước (PLCS) & & $\mathbf{0 , 8 7 9}$ \\
\hline PLCS1 & Thuế suất của thửa đất & 0,643 & 0,868 \\
\hline PLCS2 & Quy hoạch của Nhà nước & 0,737 & 0,846 \\
\hline PLCS3 & Loại đất & 0,746 & 0,845 \\
\hline PLCS4 & Giấy tờ pháp lý của thửa đất & 0,724 & 0,850 \\
\hline \multirow[t]{2}{*}{ PLCS5 } & $\begin{array}{l}\text { Các chính sách điều tiết vĩ mô của Nhà nước (đầu tư công, thu } \\
\text { hút FDI, lãi suất Ngân hàng) }\end{array}$ & 0,713 & 0,853 \\
\hline & Nhóm Yếu tố khác & & 0,792 \\
\hline K1 & Môi giới & 0,699 & 0,644 \\
\hline $\mathrm{K} 2$ & Đầu cơ đất đai & 0,608 & 0,747 \\
\hline K3 & Phong thủy & 0,601 & 0,753 \\
\hline
\end{tabular}

\subsubsection{Phân tích nhân tố khám phá EFA}

Bảng 3 và Bảng 4 liệt kê các giá trị riêng được kết hợp với mỗi hệ số tuyến tính trước khi trích xuất, sau khi trích xuất và sau khi xoay nhân tố. Kết quả phân tích thể hiện trong Bảng 3 và Bảng 4 cho thấy các thông số đều thỏa mãn điều kiện trong phân tích yếu tố khám phá EFA với: KMO nằm trong đoạn 0,5 $<0,792<1,0$; Bartlett's Sig. $=0,000<0,05$; Hệ số Eigenvalue có 6 nhóm yếu tố có giá trị Eigenvalue $>1$; Tổng phương sai giải thích của 6 nhóm yếu tố bằng $64,21 \%>50 \%$. Bảng 4 cho thấy yếu tố đầu tiên chiếm lượng phương sai chung lớn nhất
(18,044\%), đại diện giá trị riêng là 4,872. Mỗi yếu tố tiếp theo giải thích một phần của phương sai còn lại cho đến khi đạt đến một điểm mà các yếu tố đó không còn đóng góp vào mô hình. Tại thời điểm này, những yếu tố có giá trị Eigenvalue $>1$ thể hiện sự cần thiết để mô tả các kích thước cơ bản của dữ liệu. Trong nghiên cứu này, yếu tố thứ 6 , với phương sai được giải thích là 6,174 và Eigenvalues là 1,667 . Còn lại tất cả các yếu tố có Giá trị Eigenvalue $<1$ không đóng góp và không đủ số lượng cho mô hình được đưa vào. Điều này cho biết chúng không tương quan với nhau. Do đó, chỉ có 6 yếu tố (1-6) đóng góp vào mô hình. 
Bảng 3. Kiểm định KMO and Bartlett's Test

\begin{tabular}{llr}
\hline Kaiser-Meyer-Olkin Measure of Sampling Adequacy & 0,792 \\
\hline Bartlett's Test of Sphericity & Approx. Chi-Square & 3837,734 \\
& df & 351 \\
& Sig. & 0,000 \\
\hline
\end{tabular}

Bảng 4. Tổng phương sai giải thích của các nhân tố ảnh hưởng giá đất

\begin{tabular}{|c|c|c|c|c|c|c|c|c|c|}
\hline \multirow{2}{*}{$\begin{array}{c}\text { Thành } \\
\text { phần }\end{array}$} & \multicolumn{3}{|c|}{ Giá trị Eigenvalue } & \multicolumn{3}{|c|}{$\begin{array}{l}\text { Tổng bình phương của hệ số } \\
\text { tải nhân tố được trích }\end{array}$} & \multicolumn{3}{|c|}{$\begin{array}{c}\text { Tổng bình phương của hệ số } \\
\text { tải nhân tố xoay }\end{array}$} \\
\hline & Tống & $\begin{array}{r}\% \text { Phương } \\
\text { sai }\end{array}$ & 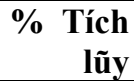 & Tổng & $\begin{array}{r}\% \text { Phương } \\
\text { sai }\end{array}$ & $\begin{array}{r}\text { \% Tích } \\
\text { lũy }\end{array}$ & Tổng & $\begin{array}{r}\% \text { Phương } \\
\text { sai }\end{array}$ & $\begin{array}{r}\text { \% } \begin{aligned} \text { Tích } \\
\text { lũy }\end{aligned}\end{array}$ \\
\hline 1 & 4,872 & 18,044 & 18,044 & 4,872 & 18,044 & 18,044 & 3,993 & 14,788 & 14,788 \\
\hline 2 & & 065 & 30,109 & 3,257 & & 30,109 & 3,406 & & 27,402 \\
\hline 3 & 3,043 & & 379 & 3,043 & & 41,379 & 3,073 & 382 & 38,784 \\
\hline 4 & 2,401 & 94 & 50,273 & 2,401 & 394 & 50,273 & 2,349 & 8,701 & 47,485 \\
\hline 5 & 2,096 & 7,764 & 58,037 & 2,096 & 7,764 & 58,037 & 2,310 & 8,554 & 56,039 \\
\hline 6 & 1,667 & 6,174 & 64,210 & 1,667 & 6,174 & 64,210 & 2,206 & 8,171 & 64,210 \\
\hline 7 & 0,815 & 3,020 & 67,230 & & & & & & \\
\hline
\end{tabular}

Bảng 5. Ma trận nhân tố xoay

\begin{tabular}{|c|c|c|c|c|c|c|}
\hline \multicolumn{7}{|c|}{ Thành phần } \\
\hline & 1 & 2 & 3 & 4 & 5 & 6 \\
\hline TN5 & 0,811 & & & & & \\
\hline TN6 & 0,759 & & & & & \\
\hline TN2 & 0,756 & & & & & \\
\hline TN1 & 0,734 & & & & & \\
\hline TN4 & 0,720 & & & & & \\
\hline TN3 & 0,680 & & & & & \\
\hline TN7 & 0,676 & & & & & \\
\hline PLCS2 & & 0,845 & & & & \\
\hline PLCS3 & & 0,838 & & & & \\
\hline PLCS4 & & 0,826 & & & & \\
\hline PLCS5 & & 0,814 & & & & \\
\hline PLSC1 & & 0,772 & & & & \\
\hline XH5 & & & 309 & & & \\
\hline XH3 & & & 304 & & & \\
\hline XH4 & & & 300 & & & \\
\hline XH2 & & & 725 & & & \\
\hline XH1 & & & 718 & & & \\
\hline KT1 & & & & & & \\
\hline KT2 & & & & & & \\
\hline KT3 & & & & & & \\
\hline HT5 & & & & & 779 & \\
\hline HT4 & & & & & 755 & \\
\hline HT3 & & & & & 737 & \\
\hline HT6 & & & & & & \\
\hline K1 & & & & & & 882 \\
\hline $\mathrm{K} 2$ & & & & & & 819 \\
\hline $\mathrm{K} 3$ & & & & & & 787 \\
\hline
\end{tabular}

Kết quả thu được trong ma trận tương quan ở Bảng 5 cho thấy tất cả các biến đều có mối quan hệ thuận chiều với nhau ở mức đô cao. Hệ số tải là 0,676 đến $0,901>0,5$. Do đó, tất cả các Tải yếu tố chuẩn hóa trong mô hình là đáng tin cậy; đây là một xác nhận về tính hợp lệ của khung lý thuyết. Điểm số về mối quan hệ giữa thành phần và khả năng mang lại thu nhập hoặc sinh lời của thửa đất cho thấy mức tích cực cao nhất 0,901 . Điều này có nghĩa là khả năng mang lại thu nhập hoặc sinh lời của thửa đất là yếu tố ảnh hưởng nhiều nhất đến giá đất tại thành phố Cần Thơ. Chỉ có yếu tố KT4 vừa có hệ số tải là 0,402 và 0,679 cần loại bỏ ra khỏi nghiên cứu nhầm tránh những sai số không đáng có. Do đó, nghiên cứu có 6 nhóm yếu tố biến độc lập với 27 thành phần quan sát phù hợp trong phân tích.

Kết quả phân tích yếu tố khám phá biến phụ thuộc (GD1, GD2, GD3, GD4) cũng thỏa mãn điều kiện trong phân tích yếu tố khám phá (EFA): KMO nằm trong đoạn $0,5<0,841<1,0$, Bartlett's $\mathrm{Sig}$, $=$ $0,000<0,05$, Hệ số Eigenvalue $=3,077>1$, Tồng phương sai giải thích $=76,916 \%>50 \%$, Hệ số tải yếu tố đều lớn hơn 0,5 . Do đó, các biến phụ thuộc cũng phù hợp với nghiên cứu này.

\subsection{Phân tích hồi quy đa biến}

Trong phần này, phân tích hồi quy bội từng bước được tính toán ở mức có ý nghĩa $(\mathrm{p}=0,05)$ để xem xét những yếu tố nào có thể ảnh hưởng đến giá đất ở tại thành phố Cần Thơ 
Bảng 6. Bảng tổng hợp mô hình

\begin{tabular}{crrrrr}
\hline Model & $\mathbf{R}$ & $\mathbf{R}^{\mathbf{2}}$ & $\mathbf{R}^{\mathbf{2}}$ hiệu chỉnh & Sai số chuẩn & Durbin-Watson \\
\hline 1 & $0,785 \mathrm{a}$ & 0,617 & 0,610 & 0,625 & 1,618 \\
\hline
\end{tabular}

Kết quả chạy mô hình hồi quy xác định hệ số $\mathrm{R}^{2}$ hiệu chỉnh $=0,610$ có nghĩa là mô hình có thể giải thích được $61 \%$ cho tổng thể về mối quan hệ giữa các yếu tố ảnh hưởng đến giá đất tại địa bàn thành phố Cần Thơ. Hay nói cách khác $61,0 \%$ sự biến động của giá đất ở có thể được giải thích bằng sự biến động của 6 nhóm biến độc lập, phần còn lại
$(39,0 \%)$ được giải thích bởi các yếu tố khác ngoài mô hình và sai số ngẫu nhiên. Kết quả kiểm định Durbin-Watson cho trị số bằng 1,618 gần với hệ số 2,0 chứng tỏ không có tương quan chuỗi bậc 1 trong mô hình (Hoàng Trọng và Chu Nguyễn Mộng Ngọc, 2005).

Bảng 7. Phân tích ANOVA

\begin{tabular}{lrrrrr}
\hline & Tổng bình phương & df & Trung bình bình phương & F & Sig, \\
\hline Regression & 201,689 & 6 & 33,615 & 86,109 & $0,000 \mathrm{~b}$ \\
Residual & 125,311 & 321 & 0,390 & & \\
Total & 327,000 & 327 & & & \\
\hline
\end{tabular}

Phân tích phương sai (ANOVA) được sử dụng để kiểm tra độ tin cậy của phân tích hồi quy. Bảng 7 cho thấy giá trị $\mathrm{F}=86,109$. Kiểm định $\mathrm{F}$ rất có ý nghĩa, do đó có thể giả định rằng có mối quan hệ tuyến tính giữa các biến trong mô hình nghiên cứu.
Bên cạnh đó, giá trị Sig. <0,01 có thể kết luận rằng mô hình đưa ra phù hợp với dữ liệu thực tế. Hay nói cách khác biến độc lập có tương quan tuyến tính với biến phụ thuộc và mức độ tin cậy $99 \%$.

Bảng 8. Hệ số hồi quy đa biến

\begin{tabular}{|c|c|c|c|c|c|}
\hline \multirow{2}{*}{ Biến độc lập } & \multicolumn{2}{|c|}{ Hệ số hồi quy chưa chuẩn hóa } & Hệ số hồi quy chuẩn hóa & \multirow{2}{*}{$\mathbf{t}$} & \multirow{2}{*}{ Sig. } \\
\hline & B & Std. Error & Beta & & \\
\hline (Constant) & 0,000 & 0,034 & & 0,000 & 1.000 \\
\hline $\mathrm{TN}$ & 0,399 & 0,035 & 0,399 & 11,545 & 0,000 \\
\hline PLCS & 0,314 & 0,035 & 0,314 & 9,096 & 0,000 \\
\hline XH & 0,200 & 0,035 & 0,200 & 5,781 & 0,000 \\
\hline KT & 0,329 & 0,035 & 0,329 & 9,510 & 0,000 \\
\hline HT & 0,315 & 0,035 & 0,315 & 9,103 & 0,000 \\
\hline $\mathrm{K}$ & 0,335 & 0,035 & 0,335 & 9,690 & 0,000 \\
\hline
\end{tabular}

Hệ số hồi quy được trình bày trong Bảng 8 cho thấy một tỷ lệ tương đối cao của sự thay đồi trong giá đất có thể được giải thích bởi các biến. Theo Bảng 8 , tất cả các biến có Sig. $<0,01$. Điều này cho thấy rằng tất cả các biến đều có tác động đáng kể đến giá đất và có mối liên hệ đáng kể giữa các biến độc lập và phụ thuộc. Như vậy nhóm biến yếu tố tự nhiên, nhóm biến yếu tố khác, nhóm biến yếu tố kinh tế, nhóm biến yếu tố hạ tầng, nhóm biến yếu tố pháp lý, chính sách Nhà nước, nhóm biến yếu tố xã hội tương quan có ý nghĩa với giá đất và độ tin cậy
99\%. Thông qua Hệ số Beta Chuẩn hóa giúp xác định được tầm quan trọng của từng biến trong mô hình hồi quy hoặc mức độ ảnh hưởng của từng biến độc lập lên biến phụ thuộc. Hệ số Beta chuẩn hóa của nhóm biến yếu tố tự nhiên có giá trị cao nhất $(0,399)$, điều này ngụ ý rằng yếu tố tự nhiên có ảnh hưởng lớn nhất đến giá đất ở tại thành phố Cần Thơ so với các yếu tố còn lại, đồng thời hệ số Beta chuẩn hóa của nhóm biến yếu tố xã hội có giá trị thấp nhất $(0,200)$, ngụ ý yếu tố xã hội ít ảnh hưởng đến giá đất ở tại thành phố Cần Thơ so với các yếu tố còn lại.

Bảng 9. Vị trí quan trọng của các yếu tố

\begin{tabular}{lrrr}
\hline Nhóm yếu tố & Beta chuẩn hóa & $(\%)$ & Hạng \\
\hline Nhóm biến yếu tố tự nhiên (TN) & 0,399 & $12,86 \%$ & 1 \\
Nhóm yếu tố pháp lý, chính sách Nhà nước (PLCS) & 0,314 & $10,12 \%$ & 5 \\
Nhóm biến yếu tố xã hội (XH) & 0,200 & $6,45 \%$ & 6 \\
Nhóm biến yếu tố kinh tế (KT) & 0,329 & $10,61 \%$ & 3 \\
Nhóm biến yếu tố hạ tầng (HT) & 0,315 & $10,16 \%$ & 4 \\
Nhóm Yếu tố khác (K) & 0,335 & $10,80 \%$ & 2 \\
\hline Tồng & 1,892 & $61,00 \%$ & \\
\hline
\end{tabular}


Từ hệ số beta chuẩn hóa, chúng ta có thể đổi thành tỷ lệ phần trăm, trong đó Nhóm biến yếu tố tự nhiên (TN) đóng góp 12,86\%, Nhóm Yếu tố khác (K) đóng góp 10,80\%, Nhóm biến yếu tố kinh tế (KT) đóng góp 10,61\%, Nhóm biến yếu tố hạ tầng (HT) đóng góp 10,16\%, Nhóm yếu tố pháp lý, chính sách Nhà nước (PLCS) đóng góp 10,12\% và Nhóm biến yếu tố xã hội (XH) đóng góp 6,45\%.

\section{KẾT LUẬN}

Kết quả nghiên cứu đã xác định được có 6 nhóm yếu tố ảnh hưởng đến giá đất trên địa bàn thành phố Cần Thơ. Chúng bao gồm nhóm yếu tố tự nhiên, yếu tố khác, yếu tố kinh tế, yếu tố hạ tầng (HT), Nhóm yếu tố pháp lý, chính sách Nhà nước và yếu tố xã hội $(\mathrm{XH})$, có mối quan hệ tích cực và đáng kể giữa các yếu tố này và giá đất. Kết quả phân tích của mô hình hồi quy tuyến tính đa biến cho thấy $61,0 \%$ sự biến động của giá đất có thể được giải thích bởi sự biến động của 6 nhóm biến độc lập, trong khi phần còn lại $(39,0 \%)$ được giải thích bởi các yếu tố khác ngoài mô hình. Tại thành phố Cần Thơ, nhóm nhân tố tự nhiên (Kích thước mặt tiền, Hình dạng thửa đất, Khoảng cách đến trục đường lớn, Chiều dài, Vị trí thửa đất, Diện tích thửa đất, Khoảng cách đến trung tâm (TTTM, TTHC, nơi làm việc, bệnh viện, trường học) có ảnh hưởng nhiều nhất đến giá đất với đóng góp 12,86\%, đối với nhóm nhân tố xã hội (Mật độ dân số quanh thửa đất, Trình độ dân trí quanh thửa đất, Đô thị hóa, An ninh trật tự khu vực, Chất lượng cảnh quan, môi trường khu vực quanh thửa đất) lại ít ảnh hưởng đến giá đất với đóng góp 6,45\% so với các yếu tố còn lại. Tuy nhóm yếu tố tự nhiên có ảnh hưởng lớn đến giá đất nhưng cũng chỉ quyết định giá cao hay thấp của thửa đất chứ không phải là yếu tố làm biến động giá đất trên thị trường. Vì thế việc xác định giá đất trong điều kiện nền kinh tế thị trường là một việc không đơn giãn, cần được thực hiện một cách khoa học và cần phải được tổng hợp nghiên cứu từ nhiều yếu tố, nhóm yếu tố khác nhau để có thể đưa ra được kết quả chính xác về giá đất. Bên cạnh đó để làm sao có thể giảm bớt sự xung đột về những mâu thuẫn trong vấn đề xác định giá đất bồi thường khi Nhà nước thu hồi đất, làm sao có thể xóa bỏ hệ thống hai giá đất phụ thuộc chủ yếu vào các chính sách của Nhà nước.

\section{TÀI LIỆU THAM KHẢO}

Cronbach L. (1951). Coefficient alpha and the internal structure of tests. Psychomerika.16: 297-334.
Hair, Jr. J. F., Anderson, R. E., Tatham R. L. \& Black W. C. (1998). Multivariate Data Analysis (5th ed.). New York: Macmillan Publishing Company.

Hair, J. F., Black, W.C., Babin, B.J., Anderson. RE. \& Tatham, RL. (2006). Multivariate data analysis (6th ed.). Upper Saddle River, NJ: Pearson University Press, 899 pages.

Hoàng Trọng và Chu Nguyễn Mộng Ngọc, 2005. Phân tích dữ liệu nghiên cứu với SPSS. Nhà xuất bản Thống kê, 349 trang.

Hồ Thị Lam Trà (2005). Giá đất và cơ sở khoa học xác định giá đất. Trong Hồ Thị Lam Trà (Chủ biên). Giáo trình Định giá đất (trang 8-23). Nhà xuất bản Đại học Nông nghiệp I Hà Nội.

Likert, R. A. (1932). A technique for measurements a attitudes, Archives of Psychology, 55 pages.

Northcraft, CA. \& Small, LE. (1979). Factors Affecting Land Prices in a Rural New Jersey County. Journal of the Northeastern Agricultural Economics Council. 2: 143-151.

Norris, M., Lecavalier, L. J \& Autism Dev Disord. (2010). Evaluating the Use of Exploratory Factor Analysis in Developmental Disability Psychological Research. Journal of Autism and Developmental Disorders. 40(1): 8-20.

Nunnally, J. C., \& Bernstein, I. H. (1994). Psychometric theory (3rd ed.). New York: McGraw-Hill, 752 pages.

Phạm Phương Nam, Nguyễn Thu Hương \& Phan Thị Thanh Huyền (2019). Đánh giá các yếu tố ảnh hưởng đến giá trúng đấu giá quyền sử dụng đất ở tại thành phố Hạ Long, tỉnh Quảng Ninh. Tạp chí Khoa học Nông nghiệp Việt Nam 2019, 17(6): 493-501.

Phan Thị Thanh Huyền, Lò Thị Hồng \& Hồ Thị Lam Trà (2017). Nhân tố ảnh hưởng đến giá đất ở thành phố Điện Biên Phủ, tỉnh Điện Biên. Tạp chí Khoa học Nông nghiệp Việt Nam, 15(9): 1186-1195.

Reynolds, JE. \& Tower, DL. (1978). Factors affecting rural land prices in an urbanizing area. Review of Regional Studies. 8(3): 23-34.

Streiner, D. (2003). Starting at the beginning: an introduction to coefficient alpha and internal consistency. Journal of personality assessment. 80: 99-103.

Vu, M.H. \& Kawashima, H. (2018). Assessing the impacts of the land development system on land prices in Vietnam's metropolises. Journal of the Japanese Agricultural Systems Society. 33(4): 123-136. 